\title{
Geociências
}

\section{Espectroscopia \\ infravermelha à baixa temperatura em quartzos e ametistas com altas concentrações de $\mathrm{OH}$ e $\mathrm{H}_{2} \mathrm{O}$}

\author{
(Low-temperature infrared spectroscopy of \\ quartz and amethyst with high concentrations \\ of $\mathrm{OH}$ and $\mathrm{H}_{2} \mathrm{O}$ )
}

\section{Resumo}

O objetivo desse trabalho foi caracterizar os defeitos pontuais relacionados aos grupos $\mathrm{OH}$ e $\mathrm{H}_{2} \mathrm{O}$ em quartzos e ametistas, crescidos em cavidades do tipo geodo em basalto da Formação Serra Geral (RS), usando a espectroscopia infravermelha (IV) a $-175^{\circ} \mathrm{C}$. Em um dos cristais, os espectros foram realizados separadamente nos setores de crescimento $r\{10 \overline{1} 1\}$ e $z\{01 \overline{1} 1\}$. Na faixa entre 3800 e 3000 $\mathrm{cm}^{-1}$, os espectros apresentaram várias bandas de absorção superpostas a uma intensa banda larga. As bandas atribuídas ao defeito $\left[\mathrm{H}_{4} \mathrm{O}_{4}\right]^{0}$ foram observadas independentemente da coloração dos cristais, enquanto que as bandas atribuídas ao centro $\left[\mathrm{AlO}_{4} / \mathrm{H}\right]^{0}$ ocorreram nos quartzos translúcido e levemente amarelado (citrino). Além da intensidade da banda larga, os espectros desses cristais se caracterizaram pela existência da banda a $3595 \mathrm{~cm}^{-1}$. Ao contrário do reportado por outros autores, verificou-se que a concentração de $\mathrm{OH}$ e $\mathrm{H}_{2} \mathrm{O}$ e a coloração violeta da ametista são maiores nos setores $z$ do que nos setores $r$. Essa inversão foi discutida em função da não uniformidade das condições de crescimento, confirmada pelo tamanho das faces romboédricas do cristal. Concluiu-se que a intensidade da coloração da ametista não é afetada pelas altas concentrações de defeitos $\mathrm{OH}$ e $\mathrm{H}_{2} \mathrm{O}$.

Palavras-chave: Quartzo natural, geodo, defeitos pontuais, grupos $\mathrm{OH}$, impurezas, setores de crescimento.

Graduanda em Engenharia de Minas Universidade Federal de Pernambuco.

E-mail:milaribas@hotmail.com

\section{Adão Benvindo da Luz}

D.Sc. Engenharia Mineral, Pesquisador Titular CETEM/MCT Centro de Tecnologia Mineral E-mail:adaobluz@cetem.gov.br

\section{Abstract}

The aim of this study is to characterize OH-related defects in natural quartz and amethyst grown in geodic cavities formed during basaltic flow. For this, infrared (IR) spectroscopy at low temperature $\left(-175^{\circ} \mathrm{C}\right)$ was carried out in amethyst and quartz crystals taken from two deposits located in the State of Rio Grande do Sul in Brazil. For one specimen, IR spectra were recorded directly upon the growth sectors $\mathrm{r}\{10 \overline{1} 1\}$ and $\mathrm{z}\{01 \overline{1} 1\}$ appearing in (0001) plates. IR spectra recorded from 3800 to $3000 \mathrm{~cm}^{-1}$ showed an intense 
broad band superposed upon sharp bands assigned to $\left[\mathrm{H}_{4} \mathrm{O}_{4}\right]^{0}$ point defects. These bands were observed in all samples and growth sectors independently from the intensity of its violet color. On the other hand, sharp bands assigned to $\left[\mathrm{AlO}_{4} / \mathrm{H}\right]^{0}$ defects were observed only in translucent and pale citrine quartz and also in the colorless regions of $r$ and $\mathrm{z}$ growth sectors in amethyst. Contrarily to previously reported data, it was observed that $\mathrm{z}$ growth sectors have the highest content of OH-related defects and the darkest violet color when compared to $r$ sectors. This reversal relation was discussed taking into account the malformed habitus of the amethyst specimen probably caused by the anisotropic solution flow inside the geode. It was concluded that the amethyst color is independent of the concentration of $\mathrm{OH}$-related defects. Besides the intense broad $\mathrm{OH}$-band, the IR spectra of such crystals were characterized by the presence of a sharp band near $3595 \mathrm{~cm}^{-1}$.

Keywords: Natural quartz, amethyst, impurity content, OH-related defects, infrared spectroscopy, growth sectors.

\section{Introdução}

Os defeitos pontuais relacionados aos radicais $\mathrm{OH}^{-}$e moléculas de água ocorrem com freqüência em cristais de quartzo, natural ou sintético, e também em suas variedades de interesse gemológico, tais como ametistas e citrinos (Kats, 1962; Chakraborty \& Lehmann, 1978; Aines \& Rossman, 1984; Guzzo et al., 1997). A espectroscopia vibracional na faixa do infravermelho (IV) é o método mais utilizado para caracterizar os defeitos $\mathrm{OH}$ em minerais (Aines \& Rossman, 1984; McMillan \& Hofmeister, 1988). Na região do IV próximo, entre 6000 e $4000 \mathrm{~cm}^{-1}$, as vibrações $\mathrm{OH}$ no quartzo se manifestam através de duas bandas de absorção atribuídas às vibrações combinadas (estiramento e flexão) das moléculas de $\mathrm{H}_{2} \mathrm{O}\left(5200 \mathrm{~cm}^{-1}\right)$ e dos grupos $\mathrm{Si}-\mathrm{OH}\left(4450 \mathrm{~cm}^{-1}\right)$. Na região do IV médio, entre 3700 e $3000 \mathrm{~cm}^{-1}$, as vibrações $\mathrm{OH}$ ocorrem na forma de bandas estreitas superpostas a uma banda larga isotrópica. A posição das bandas estreitas depende da impureza presente na vizinhança dos radicais $\mathrm{OH}^{-}$; enquanto que a banda larga é conseqüência das vibrações de estiramento dos grupos $\mathrm{OH}$ e das moléculas $\mathrm{H}_{2} \mathrm{O}$. Devido à diminuição das vibrações atômicas da estrutura cristalina, a identificação das bandas estreitas torna-se mais fácil quando os espectros são realizados em temperaturas criogênicas (Aines \& Rossman, 1984).

A importância dos defeitos relacionados ao $\mathrm{OH}$ e $\mathrm{H}_{2} \mathrm{O}$ no quartzo deve-se ao fato de que, sob condições de altas temperaturas e pressões, os cristais hidratados são passíveis de deformação plástica (Griggs \& Blacic, 1965; Blacic \& Christie, 1984). Esse mecanismo, denominado enfraquecimento hidrolítico, contribuiu para explicar a plasticidade observada nas rochas da crosta terrestre. Recentemente, o mapeamento do coeficiente de absorção a $3400 \mathrm{~cm}^{-1}$ nos setores $r\{10 \overline{1} 1\}$ e $z\{01 \overline{1} 1\}$ foi utilizado para estimar a velocidade relativa de crescimento de um quartzo hialino euédrico (Ihinger \& Zink, 2000). Do ponto de vista tecnológico, as impurezas $\mathrm{OH}$ e $\mathrm{H}_{2} \mathrm{O}$ no quartzo sintético afetam o desempenho metrológico de ressonadores piezelétricos usados em osciladores de alta freqüência (Brice, 1985). Por fim, sabe-se que elevadas concentrações de $\mathrm{OH}$ e $\mathrm{H}_{2} \mathrm{O}$ comprometem a coloração dos cristais hialinos mediante altas doses de radiação gama (Guzzo et al., 1997). Logo o estudo dessas impurezas também poderá contribuir para o processamento das variedades de quartzo para finalidades gemológicas.

Em estudo anterior, foi observado que quartzos coletados dos depósitos de ametistas do Estado do Rio Grande do Sul apresentam alta concentração de $\mathrm{OH}$ e $\mathrm{H}_{2} \mathrm{O}$ (Guzzo et al., 1997). Na ocasião, apenas foram considerados espectros IV obtidos na temperatura ambiente. Como se sabe, as ocorrências de quartzos e ametistas no Rio Grande do Sul estão relacionadas aos derrames basálticos da bacia do Paraná pertencente à Formação Serra Geral. A gênese dos cristais ocorre no interior de cavidades ou zonas vesiculares formando depósitos do tipo geodo em basalto, arredondados ou ovóides, de tamanhos diver- sos. Os geodos são constituídos por uma crosta externa de celadonita e calcita, uma faixa intermediária de cristais hialinos ou translúcidos e uma faixa interna de ametistas com diferentes tonalidades. $\mathrm{Na}$ parte central, também são encontrados cristais de calcita e gipsita (Juchen, 1999; Gilg et al., 2003). Atualmente, a região do Médio Alto Uruguai no RS é a principal produtora de ametistas exportadas pelo Brasil. Assim, buscando avançar na compreensão dos mecanismos de incorpo-

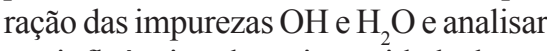
sua influência sobre a intensidade da cor violeta, o presente trabalho apresenta novas medidas de IV obtidas à $-175^{\circ} \mathrm{C}$ em cristais translúcidos e ametistas de duas ocorrências distintas. Embora se saiba que a coloração da ametista devese à incorporação de impurezas férricas e à disponibilidade de radiação ionizante no local de crescimento (Lehmann, 1975; Balitsky et al., 1986), a incorporação de $\mathrm{OH}$ e $\mathrm{H}_{2} \mathrm{O}$ nos setores de crescimento é ainda pouco conhecida para as ametistas daquela região.

\section{Materiais e Métodos}

Para esse estudo foram utilizados duas ametistas, dois quartzos translúcidos e um cristal levemente amarelado (citrino), procedentes do município de Ametista do Sul (RS) e do distrito de Morro Cristal (Caxias do Sul, RS). Como mostra a Figura 1, os cristais são de pequenas dimensões, desprovidos das faces prismáticas $m$ e com as faces piramidais $r$ e $z$ com tamanhos aproximadamente iguais. Apenas a região terminal dos cristais é transparente.

Lâminas de aproximadamente 2,0 mm de espessura foram cortadas de forma perpendicular ao eixo c (eixo óptico) e lapidadas sobre placas de vidro com grãos de alumina de granulometria decrescente. Em seguida, as amostras foram limpas em banho de acetona e, então, polidas opticamente com uma suspensão de alumina de $1 \mu \mathrm{m}$ sobre disco rotativo com feltro. Após o polimento, observou-se que os setores de crescimento $r\{10 \overline{1} 1\}$ e $z\{01 \overline{1} 1\}$ aparecem com grande definição em uma das amostras 
de Ametista do Sul, conforme mostrado na Figura 2(a). A identificação dos setores foi efetuada pela visualização das franjas de Brewster com estereomicroscópio usando luz polarizada. Essas franjas, cujo detalhe é mostrado na Figura 2(b), se concentram, principalmente, nos setores $r$ e correspondem às fronteiras da estrutura de domínios dos geminados que seguem a Lei do Brasil (Schlossin \& Lang, 1965; McLaren \& Pitkethly, 1982). Segundo Sunagawa (2005), esses geminados ocorrem, preferencialmente, nos quartzos crescidos a baixas temperaturas.
A Tabela 1 apresenta a concentração das principais impurezas metálicas das ametistas e quartzos utilizados nesse estudo. Tais concentrações foram determinadas anteriormente por Pasquali (1992), utilizando a espectrometria de absorção atômica. A análise das impurezas foi realizada em fragmentos de cada cristal, após ocorrer a remoção das inclusões fluidas por meio de choque térmico e lixiviação aquosa. Tais procedimentos buscaram eliminar a interferência dos cátions encontrados na solução das inclusões fluidas $\left(\mathrm{Na}^{+}, \mathrm{K}^{+}, \mathrm{Ca}^{2+}, \mathrm{Mg}^{2+}, \mathrm{Li}^{+}\right.$, $\mathrm{Al}^{3+}, \mathrm{Fe}^{3+}$ ) sobre a concentração das im- purezas estruturais (substitucionais ou intersticiais) dos cristais.

Os espectros IV foram obtidos com um espectrômetro Perkin Elmer 580B de duplo feixe à temperatura de aproximadamente $-175^{\circ} \mathrm{C}$. Para isso, cada amostra foi colocada em um criostato contendo nitrogênio líquido. O vácuo e a estabilização da temperatura foram atingidos antes do início da medida, realizada entre 3800 e $2500 \mathrm{~cm}^{-1}$. As janelas da célula criostática permaneceram aquecidas durante o experimento. $\mathrm{O}$ feixe foi limitado com uma janela suplementar de $4 \mathrm{~mm}$ de diâmetro e os espectros foram obtidos

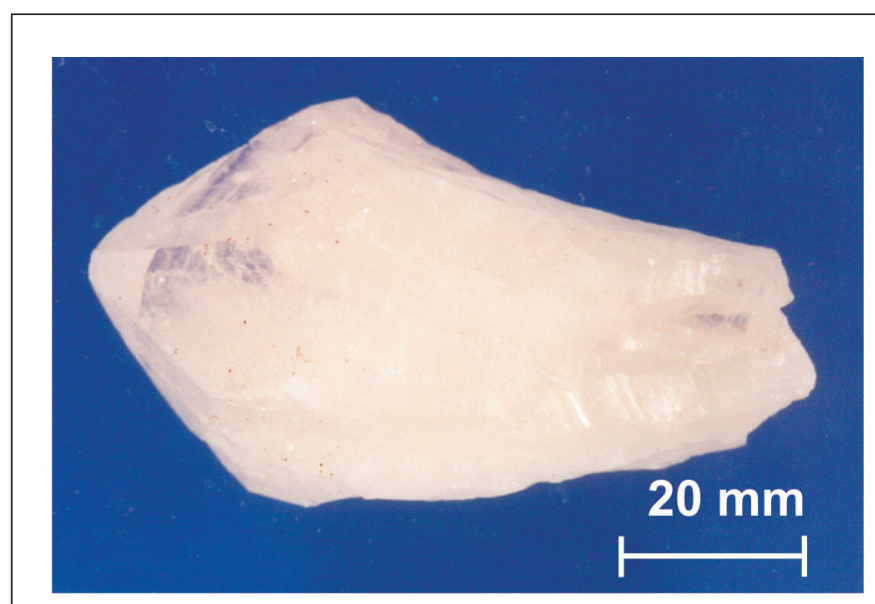

(a) quartzo translúcido

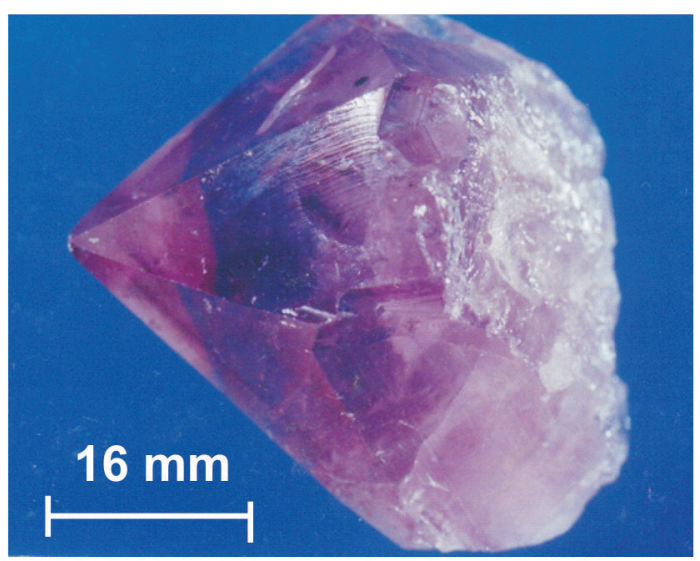

(b) ametista

Figura 1 - Cristais procedentes de Ametista do Sul (RS).

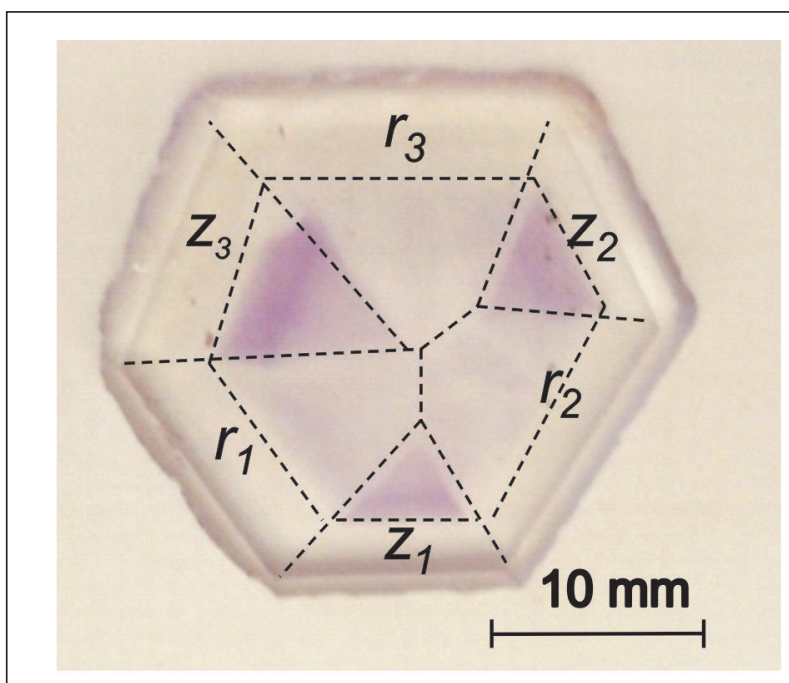

(a) placa (0001)

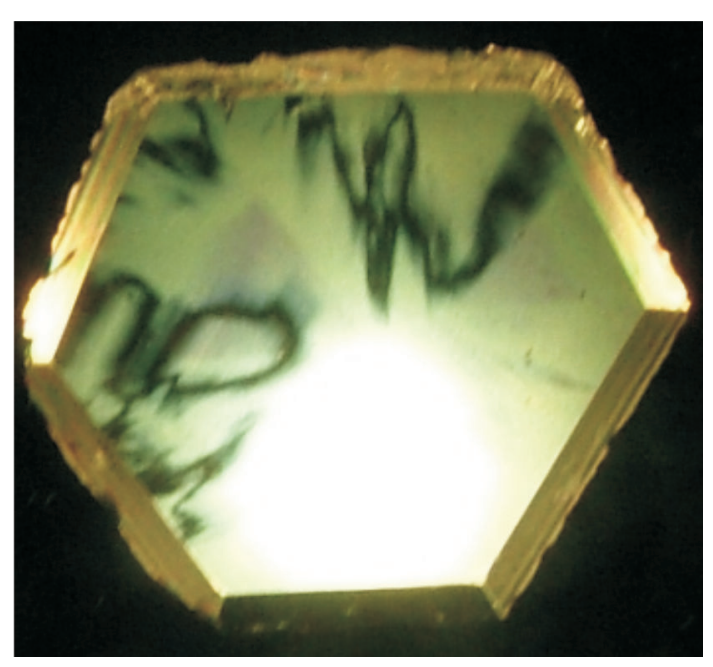

(b) franjas de Brewster

Figura 2 - Placa de ametista mostrando os setores de crescimento e franjas de Brewster partindo dos setores $r$. 
Espectroscopia infravermelha à baixa temperatura em quartzos e ametistas com altas concentrações de $\mathrm{OH}$ e $\mathrm{H}_{2} \mathrm{O}$

Tabela 1 - Impurezas metálicas associadas à estrutura dos cristais.

\begin{tabular}{|c|c|c|c|c|c|c|}
\hline & \multicolumn{6}{|c|}{ Concentração de impurezas $\left(\mathrm{N} / 10^{6} \mathrm{Si}\right)$} \\
\hline & $\mathrm{Al}$ & $\mathrm{Fe}$ & $\mathrm{Li}$ & $\mathrm{Na}$ & $\mathrm{K}$ & $\mathrm{Ca}$ \\
\hline Limite de detecção & 0,05 & 0,01 & 0,001 & 0,001 & 0,002 & 0,002 \\
\hline SG-translúcido* & 37,5 & 5,6 & 3,7 & 45,7 & 9,5 & - \\
\hline MC-translúcido** & 56,0 & 10,7 & 14,0 & 49,0 & 31,1 & 42,8 \\
\hline MC-esfumaçado** & 37,2 & 18,1 & 7,8 & 49,6 & 28,9 & 43,6 \\
\hline MC-ametista** & 31,1 & 63,8 & 12,6 & 54,1 & 44,3 & 162,2 \\
\hline SG-ametista** & 35,2 & 30,5 & 13,9 & 39,5 & 10,9 & 4,0 \\
\hline
\end{tabular}

* Guzzo et al., 1997

**Pasquali, 1992

- não determinado

no modo de transmitância. Espectros adicionais foram obtidos à temperatura ambiente. $O$ coeficiente de absorção decimal $(\alpha)$ das bandas foi calculado pela equação de Beer-Lambert (Paterson, 1982), ou seja :

$\alpha=\left[\log \left(T_{3800} / T_{x}\right)\right] / t$

onde $t$ é a espessura da amostra (cm), $T_{3800}$ é o valor da transmitância na posição de referência $\left(3800 \mathrm{~cm}^{-1}\right)$ e $T_{x}$ é o valor da transmitância na extremidade da banda, ou seja, na posição que corresponde ao máximo de absorção. A influência da banda larga sobre a intensidade das bandas estreitas foi minimizada subtraindo-se o valor da intensidade de absorção em $3500 \mathrm{~cm}^{-1}$, sendo, então, obtida a intensidade relativa de absorção para cada banda. A escolha da absorção a $3500 \mathrm{~cm}^{-1}$ deve-se ao fato de que, nessa região, não ocorrerem bandas estreitas de absorção. Por sua vez, usando a calibração proposta por Paterson (1982), a concentração de $\mathrm{OH}$ e $\mathrm{H}_{2} \mathrm{O}$ de cada amostra foi calculada pela integração numérica da banda larga, da seguinte forma (Guzzo et al., 1997):

$C_{B L}=(1 / 50) \sum\left\{\left[\log \left(T_{b}(w) / T_{a}(w)\right) / t\right] /(3780-w)\right\} \Delta w$

onde $C_{B L}$ é a concentração de $\mathrm{OH}$ e $\mathrm{H}_{2} \mathrm{O}$ associada à banda larga $(\mathrm{mol} \mathrm{H} / \mathrm{l}), w$ é o número de onda $\left(\mathrm{cm}^{-1}\right), t$ é a espessura da amostra $(\mathrm{cm}), \Delta w$ é o intervalo de integração $\left(40 \mathrm{~cm}^{-1}\right), T_{b}$ é a transmitância na linha de base (\%) e $T_{a}$ é a transmitância na linha de absorção (\%). A concentração de $\mathrm{OH}_{\mathrm{H}} \mathrm{H}_{2} \mathrm{O}$ foi realizada utilizando os espectros obtidos a $-175^{\circ} \mathrm{C}$. Segundo Paterson (1982), o erro associado a essa determinação é da ordem de $30 \%$.

\section{Resultados e Discussão}

A Figura 3 apresenta os espectros IV dos setores $r$ e $z$ da amostra de ametista apresentada na Figura 2(a). Na Figura 3(a), observa-se que cada espectro obtido à temperatura ambiente é constituído por uma intensa banda larga sobre a qual está superposta uma banda estreita a $3585 \mathrm{~cm}^{-1}$. Essa banda foi atribuída ao defeito denominado as-grown $\mathrm{OH}$ observado em cristais sintéticos obtidos por crescimento hidrotérmico em soluções de $\mathrm{NaOH}$ ou $\mathrm{Na}_{2} \mathrm{Co}_{3}$ (Lipson \& Kahan, 1985). Atualmente, a estrutura aceita, para esse defeito, é o centro $\left[\mathrm{H}_{4} \mathrm{O}_{4}\right]^{0}$, formado pela substi- tuição do íon $\mathrm{Si}^{4+}$ por quatro prótons $\mathrm{H}^{+}$, originalmente proposto por Nuttall e Weil (1980). As bandas de absorção a 3300 e $3200 \mathrm{~cm}^{-1}$, atribuídas aos harmônicos das vibrações Si-O (Kats, 1962), não são claramente observadas nos espectros das regiões incolores devido à intensa banda larga. Os sinais presentes a 3612 e $3595 \mathrm{~cm}^{-1}$ ocorrem, respectivamente, nas regiões coloridas dos setores $r \mathrm{e}$ $z$, delimitas por linhas tracejadas na Figura 2(a). Os centros de defeitos responsáveis por essas bandas ainda não são conhecidos.

Os espectros obtidos à baixa temperatura (Figura 3(b)) mostram maior diferença entre os setores de crescimento da ametista. Nota-se de imediato que a banda a $3438 \mathrm{~cm}^{-1}$ do espectro do setor $r$ à temperatura ambiente foi decomposta em duas bandas estreitas e que a intensidade da banda larga ainda é maior para as regiões incolores dos setores $r$ e $z$. As bandas de absorção observadas a 3579 , 3437,3394 e $3349 \mathrm{~cm}^{-1}$ são atribuídas ao defeito $\left[\mathrm{H}_{4} \mathrm{O}_{4}\right]^{0}$, sendo que a banda a $3579 \mathrm{~cm}^{-1}$ corresponde àquela observada a $3585 \mathrm{~cm}^{-1}$ à temperatura ambiente. Além do quartzo sintético crescido em solução convencional $(\mathrm{NaOH}$ ou $\mathrm{Na}_{2} \mathrm{Co}_{3}$ ), as bandas atribuídas ao defeito $\left[\mathrm{H}_{4} \mathrm{O}_{4}\right]^{0}$ foram observadas em cristais crescidos em solução de $\mathrm{KCl}$ ou em água pura (Hosaka et al., 1981). Observa-se que os espectros da região colorida do setor $r$, obtidos a temperatura ambiente e à $-175^{\circ} \mathrm{C}$, são semelhantes àqueles do quartzo crescido em solução de $\mathrm{KCl}$. Porém os cristais sintéticos não apresentaram a banda a $3595 \mathrm{~cm}^{-1}$, claramente separada da banda a $3585 \mathrm{~cm}^{-1}$ no espectro do setor $z$ da ametista obtido à baixa temperatura. Conforme reportado anteriormente por Kats (1962), as bandas que ocorrem em maiores números de onda (maior energia) sofrem menor efeito da temperatura. Por esse motivo, a banda a $3595 \mathrm{~cm}^{-1}$ ocorre na mesma posição em ambas as temperaturas investigadas. Por meio da eletrodifusão, Kats (1962) realizou a troca de íons $\mathrm{Li}^{+}$por K $\mathrm{K}^{+}$ em um cristal hialino e verificou que novas bandas de absorção aparecem a 3610 e $3585 \mathrm{~cm}^{-1}$. Portanto é possível que a banda observada a $3595 \mathrm{~cm}^{-1}$, nas 
Pedro Luiz Guzzo et al.

regiões coloridas dos setores $r$ e $z$, esteja relacionada a defeitos envolvendo radicais $\mathrm{OH}^{-}$e íons $\mathrm{K}^{+}$. A presença de $\mathrm{K}$ nesta e nas demais amostras fortalece essa hipótese.

A Figura 4 apresenta espectros IV de quartzos procedentes de Ametista do Sul (SG) e Morro Cristal (MC). Os espectros têm características semelhantes àqueles apresentados na Figura 3(b), com a presença das bandas associadas ao defeito $\left[\mathrm{H}_{4} \mathrm{O}_{4}\right]^{0}$. No espectro do quartzo levemente amarelado (citrino), também ocorrem as bandas a 3364 e $3305 \mathrm{~cm}^{-1}$, típicas dos cristais hialinos na condição natural e dos cristais sintéticos irradiados com raios $\gamma$ ou raios $\mathrm{X}$ (Halliburton, 1985). Trata-se das bandas atribuídas ao centro $\left[\mathrm{AlO}_{4} / \mathrm{H}\right]^{0}$, resultante da compensação de carga efetuada pelo próton $\mathrm{H}^{+}$ vizinho ao $\mathrm{Al}^{3+}$ substitucional (Kats, 1962; Halliburton, 1985). Como se sabe, a coloração esfumaçada do quartzo irradiado está relacionada com a ocorrência dos centros paramagnéticos $\left[\mathrm{AlO}_{4}\right]^{0}$ (O’Brien, 1955; Halliburton, 1985). Embora com menor intensidade, a banda a $3595 \mathrm{~cm}^{-1}$ também ocorre nos cristais translúcidos e no citrino, sugerindo a incorporação de íons $\mathrm{K}^{+}$.

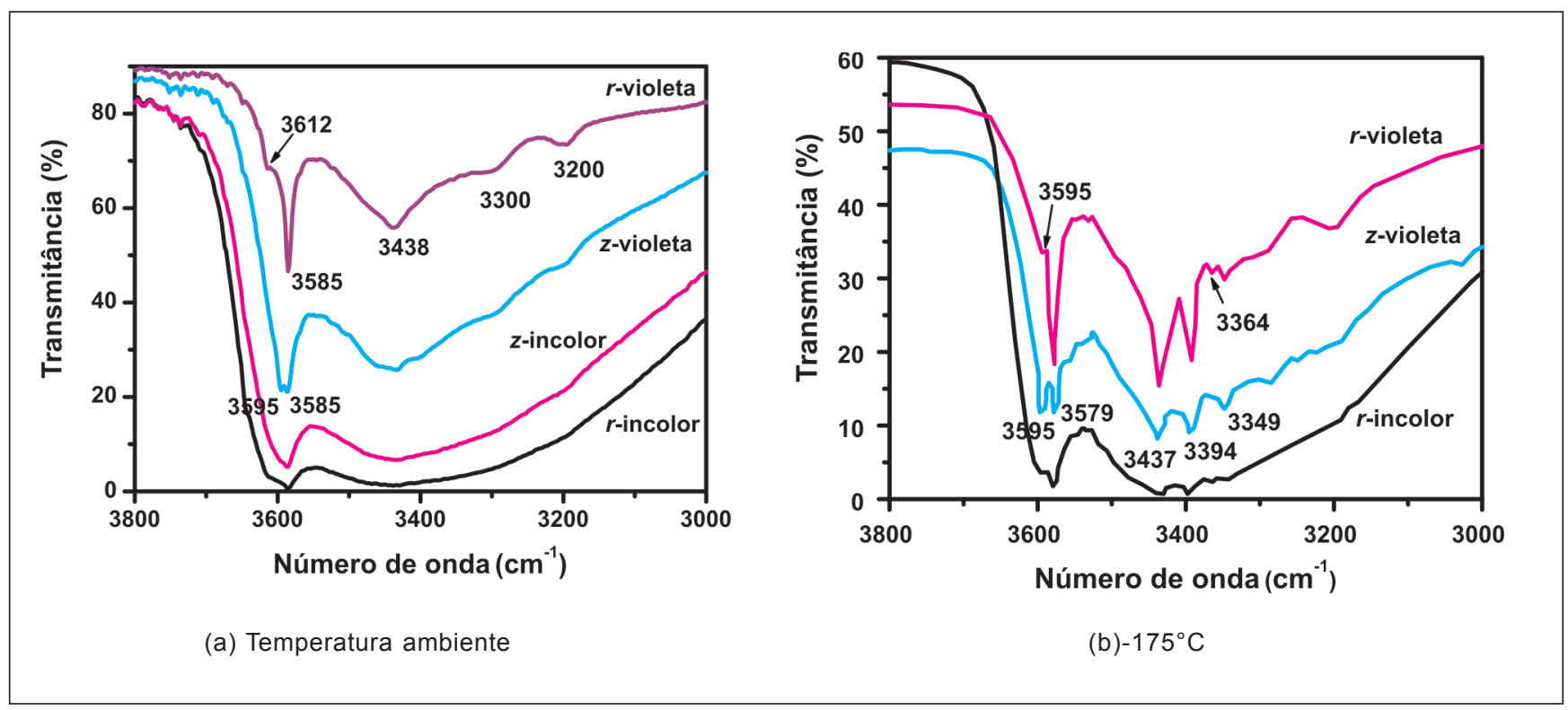

Figura 3 - Espectros IV dos setores de crescimento observados no plano (0001) de uma amostra de Ametista do Sul (RS).

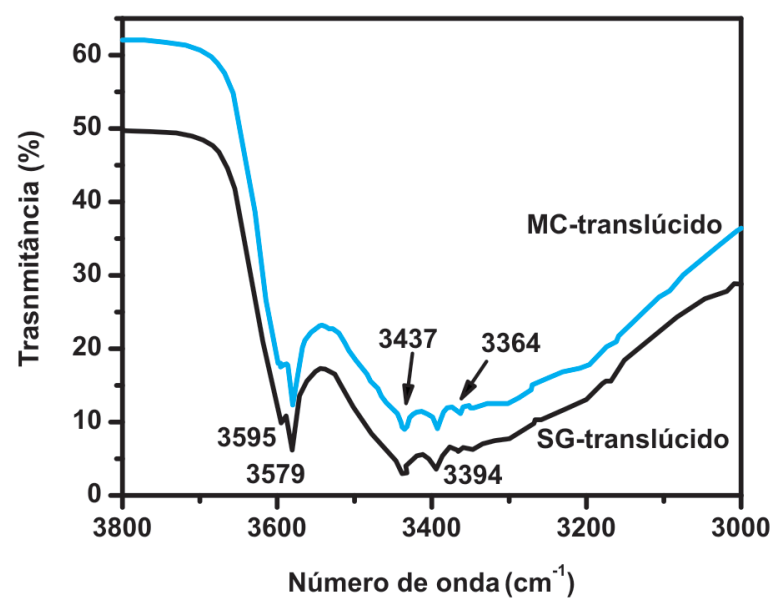

(a) Quartzo translúcido

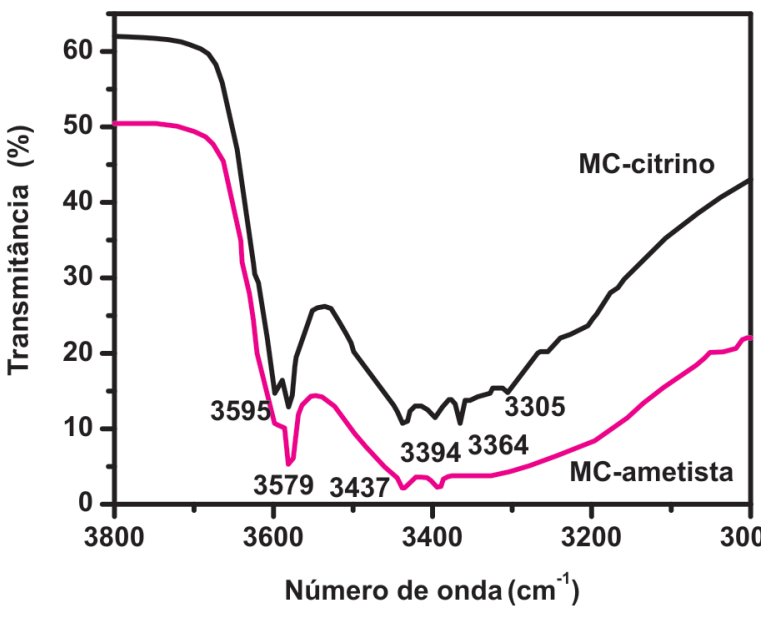

(b) Citrino e ametista

Figura 4 - Espectros IV obtidos a $-175^{\circ} \mathrm{C}$ em placas (0001) de quartzos translúcido, citrino e ametista do Rio Grande do Sul. 
A Tabela 2 apresenta os coeficientes de absorção das bandas identificadas nas Figuras 3 e 4. N Tabela 2, também são apresentados os valores de $\alpha_{3500}$ e das concentrações dos grupos $\mathrm{OH}$ e $\mathrm{H}_{2} \mathrm{O}$. Comparando os coeficientes de absorção dos setores $r$ e $z$, observase que a intensidade das bandas atribuídas ao defeito $\left[\mathrm{H}_{4} \mathrm{O}_{4}\right]^{0}$ é maior nas regiões de tonalidade violeta dos setores $r$, cristalizadas após as regiões incolores (externas). Ao mesmo tempo, essas regiões apresentam menores concentrações de $\mathrm{OH}$ e $\mathrm{H}_{2} 0$ do que as regiões violetas dos setores $z$ e incolores de ambos os setores. Logo conclui-se que a incorporação do defeito $\left[\mathrm{H}_{4} \mathrm{O}_{4}\right]^{0}$ ocorre tardiamente e preferencialmente nos setores $r$. No caso dos setores $\mathrm{z}$, a espectroscopia IV revelou menor diferenciação entre a incorporação dos defeitos $\left[\mathrm{H}_{4} \mathrm{O}_{4}\right]^{0} \mathrm{e}$ grupos $\mathrm{OH}_{\text {e }} \mathrm{H}_{2} \mathrm{O}$. Comparado aos coeficientes de absorção dos outros cristais, nota-se que a coloração violeta das regiões internas dos setores $z$ não está relacionada com a intensidade das bandas $\left[\mathrm{H}_{4} \mathrm{O}_{4}\right]^{0}$ e tampouco com a banda larga isotrópica. Além disso, como a banda de absorção a $3595 \mathrm{~cm}^{-1}$ ocorre nos cristais translúcido e citrino, o defeito associado a essa banda não pode ser o único responsável pela coloração violeta da ametista. Por fim, a Tabela 2 mostra a incompatibilidade da coloração violeta com a presença do $\mathrm{Al}^{3+}$ substitucional, ou seja, as bandas associadas ao centro $\left[\mathrm{AlO}_{4} / \mathrm{H}\right]^{0}$ não são observadas na ametista de Morro Cristal e nas regiões violetas dos setores $z$ da ametista de Ametista do Sul. A incompatibilidade da coloração violeta com os centros de $\mathrm{Al}$ é confirmada pelas menores concentrações de $\mathrm{Al}$ detectadas nas ametistas. Observando-se a Tabela 1, nota-se, também, que a concentração de $\mathrm{Fe}$ em relação à de Al, i.e., a razão entre as duas concentrações, é, substancialmente, superior para as ametistas, em particular para a amostra de Morro Cristal.

Espectros IV nos setores de crescimento da ametista foram apresentados por Kats (1962) e depois por Balitsky e Balitskaya (1986). Utilizando uma ametista natural, Kats (1962) observou que maiores bandas largas e menores bandas estreitas ocorrem, simultaneamente, nos setores de intensa coloração violeta de uma ametista natural. Porém Kats (1962) não cita a natureza dos setores. Por sua vez, Balitsky e Balitskaya (1986) observaram que, em ametista cultivada em solução alcalina de $\mathrm{K}_{2} \mathrm{CO}_{3}$ contendo ferro, as colorações mais intensas e as maiores bandas largas ocorrem, simultaneamente, nos setores $r$. Trabalhos anteriores também relatam que os setores $r$ de ametistas naturais apresentam colorações mais intensas e maiores concentrações de ferro do que nos setores $z$ (Schlossin \& Lang, 1965; Mclaren \& Pitkethly, 1982). Infelizmente, as concentrações de impurezas da Tabela 1 não permitem uma análise detalhada da distribuição do ferro nos setores de crescimento da amostra de Ametista do Sul.

Quando se faz comparação com os trabalhos citados, percebe-se que as tonalidades de violeta na Figura 2(a) e os coeficientes de absorção da Tabela 2 mostram uma inversão nas propriedades dos setores $r$ e $z$ da amostra investigada. A hipótese de que a incorporação das impurezas $\mathrm{Fe}, \mathrm{OH}$ e $\mathrm{H}_{2} \mathrm{O}$ tenha ocorrido de forma inversa está baseada no fato de que o tamanho das faces naturais e a

Tabela 2 - Coeficientes de absorção (relativos à absorção a $3500 \mathrm{~cm}^{-1}$ ) das bandas atribuídas aos defeitos $\left[\mathrm{H}_{4} \mathrm{O}_{4}\right]^{0}$ e $\left[\mathrm{AlO} \mathrm{O}_{4} / \mathrm{H}\right]^{0}$ medidos nos espectros obtidos a $-175^{\circ} \mathrm{C}$.

\begin{tabular}{|c|c|c|c|c|c|c|c|c|}
\hline Defeito & \# & \multicolumn{4}{|c|}{$\left[\mathrm{H}_{4} \mathrm{O}_{4}\right]^{0}$} & {$\left[\mathrm{AlO}_{4} / \mathrm{H}\right]^{0}$} & \multicolumn{2}{|c|}{$\mathrm{OH}+\mathrm{H}_{2} \mathrm{O}$} \\
\hline $\mathrm{N}^{\circ}$ de onda $\left(\mathrm{cm}^{-1}\right)$ & 3595 & 3579 & 3437 & 3394 & 3349 & 3364 & \multirow{2}{*}{$\begin{array}{l}\alpha_{3500} \\
\left(\mathrm{~cm}^{-1}\right)\end{array}$} & \multirow{2}{*}{$\begin{array}{c}C_{B L} \\
\left(\mathrm{~N} / 10^{6} \mathrm{Si}\right)\end{array}$} \\
\hline Amostra & \multicolumn{6}{|c|}{$\left(\alpha_{w}-\alpha_{3500}\right) / \alpha_{3500}$} & & \\
\hline SG-translúcido & 0,08 & 0,40 & 0,89 & 0,76 & 0,36 & 0,36 & 3,41 & 2625 \\
\hline MC-translúcido & 0,04 & 0,33 & 0,59 & 0,58 & - & 0,42 & 2,77 & 2198 \\
\hline MC-esfumaçado & 0,27 & 0,39 & 0,53 & 0,49 & * & 0,55 & 2,46 & 1921 \\
\hline MC-ametista & * & 0,37 & 0,91 & 0,88 & - & - & 7,18 & 6282 \\
\hline SG-ametista $r(v)$ & * & 1,32 & 1,70 & 1,25 & 0,27 & 0,20 & 0,91 & 679 \\
\hline SG-ametista $r(\mathrm{i})$ & * & 0,48 & 0,88 & 0,88 & - & 0,37 & 4,66 & 3608 \\
\hline SG-ametista $z(v)$ & 0,45 & 0,45 & 0,83 & 0,72 & 0,41 & - & 1,90 & 1445 \\
\hline
\end{tabular}

- banda não observada

(v) violeta

(i) incolor

* banda observada, mas com pequena intensidade

\# defeito não identificado 
morfologia dos setores podem ser alterados durante o crescimento dos cristais naturais em virtude de variações de pressão, temperatura, concentração de impurezas ou da direção das correntes da solução mineralizante (Sunagawa, 2005). No crescimento hidrotérmico de ametistas sintéticas, Balitsky et al. (2001) mostraram que o tamanho e a coloração dos setores $r$ e $z$ variam em função da posição da semente em relação à direção do fluxo da solução mineralizante. $\mathrm{Na}$ Figura 1(b), ao contrário do que geralmente é observado no quartzo hialino, as faces romboédricas da ametista ocorrem sem diferença perceptível de tamanho, evidenciando uma alteração no hábito prismático-piramidal do quartzo. Essa distorção no hábito cristalino e a diferença de tamanho entre setores de mesma natureza observadas da Figura 2(a) sugerem que o cristal em questão se desenvolveu em um ambiente anisotrópico, ou seja, fora das condições de crescimento onde prevaleceriam as formas de equilíbrio do cristal. Pela Figura 2(a), nota-se, também, que a anisotropia do ambiente de crescimento diminuiu no estágio final de crescimento, pois a diferença de tamanho entre os setores $r$ e $z$ é maior na região central do cristal, onde a tonalidade violeta é mais intensa. A diferença de tonalidade dentro de um mesmo setor é explicada pela expulsão dos íons $\mathrm{Fe}^{3+}$ da interface de crescimento para a solução. $\mathrm{O}$ mesmo não ocorre para as impurezas $\mathrm{OH}$ e $\mathrm{H}_{2} \mathrm{O}$ que são incorporadas durante todos os estágios de crescimento; porém em maior quantidade na fase inicial, quando são cristalizadas as regiões incolores dos respectivos setores. A incorporação diferenciada dos íons $\mathrm{Fe}^{3+}$ poderá ser confirmada quando a concentração de ferro for analisada nos diferentes setores de crescimento da amostra.

\section{Conclusão}

A espectroscopia infravermelha à baixa temperatura confirmou a presença de grandes concentrações de defeitos $\mathrm{OH}$ e $\mathrm{H}_{2} \mathrm{O}$ em quartzos crescidos em geodos, independentemente da coloração do cristal. A presença da banda de absorção a $3595 \mathrm{~cm}^{-1}$, cuja natureza pode estar relacionada a íons $\mathrm{K}^{+}$na vizinhança dos radicais $\mathrm{OH}^{-}$, e a ausência das bandas a 3367 e $3306 \mathrm{~cm}^{-1}$, relacionadas ao defeito $\left[\mathrm{AlO}_{4} / \mathrm{H}\right]^{0}$ e observadas com maior intensidade no citrino, consistem nas principais características que diferenciam os espectros IV das ametistas. Constatou-se, também, que a concentração de ferro em relação à de alumínio é maior nos cristais de ametista. Contrariamente ao observado nos cristais esfumaçados e irradiados artificialmente, onde o grau de escurecimento diminui com a concentração de grupos $\mathrm{OH}$ e $\mathrm{H}_{2} \mathrm{O}$, a coloração violeta não é afetada pela concentração de $\mathrm{OH}$ e $\mathrm{H}_{2} \mathrm{O}$.

Os espectros IV dos setores de crescimento da ametista analisada mostraram que a incorporação de defeitos $\mathrm{OH}$ e $\mathrm{H}_{2} \mathrm{O}$ assim como a intensidade violeta, ocorrem, preferencialmente, nos setores $z$. Esse resultado, oposto ao relatado por outros autores, confirma que a partição das impurezas durante o crescimento do cristal é sensível ao ambiente onde o mesmo se desenvolve. Por sua vez, os setores $r$ apresentaram menor coloração violeta e maior intensidade das bandas atribuídas ao defeito $\left[\mathrm{H}_{4} \mathrm{O}_{4}\right]^{0}$.

\section{Agradecimentos}

O autor Pedro Luiz Guzzo agradece à Dra . Maria A. Pasquali pela coleta dos cristais e aos Professores Henry Merigoux e Pierre Zecchini (Université de Franche-Comté, Besançon, França) pelo uso do espectrômetro IV. Milena Ribas de Miranda agradece ao programa PIBIC/CNPq/CETEM pela bolsa de iniciação científica.

\section{Referências bibliográficas}

AINES, R.D., ROSSMAN, G.R. Water in minerals? A peak in the infrared. $\boldsymbol{J}$. Geophys. Res., v. 89, p.4059-4071, 1984.

BALITSKY, V.S., BALITSKAYA, O.V. The amethyst-citrine dichromatism in quartz and its origin. Phys. Chem. Miner., v. 13, p. 415-421, 1986.

BALITSKY, V.S., IWASAKI, H., SUNAGAWA, I. Growth morphologies and their computer simulations in quartz crystals synthesized under various growth conditions. In : Proc. of The $13^{\text {th }}$ International Conference on Crystal Growth, 2001, Kyoto, Anais...,v. 1, p.345.

BLACIC, J.D., CHRISTIE, J.M. Plasticity and hydrolytic weakening of quartz single crystals. J. Geophys. Res., v. 89, p.4223-4239, 1984.

BRICE, J.C. Crystals for quartz resonators. Reviews of Modern Physics, v. 57, n. 1, p. 105-146, 1985.

CHAKRABORTY, D., LEHMANN, G. On the fine structure in the infrared spectra of clear natural quartz, amethyst, citrine and synthetic quartz crystals in the 3400 $\mathrm{cm}^{-1}$ region. $\boldsymbol{Z}$. Naturforsch., v. 33a, p. 290-293, 1978.

GILG, H.A., MORTEANI, G., KOSTITSYN, Y., PREINFALK, C., GATTER, I., STRIEDER, A.J. Genesis of amethyst geodes in basalt rocks of the Serra Geral Formation (Ametista do Sul, Rio Grande do Sul, Brazil): a fluid inclusion, REE, oxygen, carbon and $\mathrm{Sr}$ isotope study on basalt, quartz and calcite. Mineralium Depostita, v. 38, p. 1009-1025, 2003.

GRIGGS, D.T., BLACIC, J.D. Anomalous weakness of synthetic crystals. Science, v. 147, p.292-295, 1965.

GUZZO, P.L., IWASAKI, F., IWASAKI, H. Al-related centers in relation to $\gamma$ irradiation response in natural quartz. Phys. Chem. Miner., v. 24, p.254-263, 1997.

HALLIBURTON, L.E. Defect models and radiation damage mechanisms in alphaquartz. Cryst. Latt. Def. and Amorph. Mat., v. 12, p.163-190, 1985.

HOSAKA, M., TAKI, S., NAGAI, K., ASAHARA, J. Synthetic quartz crystals grown in $\mathrm{NaCl}, \mathrm{KCl}$ solutions and pure water, and their low temperature infrared absorption. In: Proc. $35^{\text {th }}$ Ann. Symp. on Freq. Control, 1981, Ft. Monmouth, Anais...,v. 1, p.304-311.

IHINGER, P. D., ZINK, S. I. Determination of relative growth rates of natural quartz crystals. Nature, v. 404, p.865-869, 2000.

JUCHEM, P. L. Minerologia, geologia $\boldsymbol{e}$ gênese dos depósitos de ametista da região do Alto Uruguai, Rio Grande do 
Sul. São Paulo: IGc-USP, 1999. 225p.

(Tese de Doutorado).

KATS, A. Hydrogen in alpha-quartz. Philips

Res. Repts., v.17, p.113-195, 201-279, 1962.

LEHMANN, G. On the color centers of iron in amethyst and synthetic quartz: a discussion. Am. Mineral., v. 60, p. 335 337, 1975.

LIPSON, H.G., KAHAN, A. Infrared characterization and hydrogen defect centers in irradiated quartz. J. Appl. Phys., v. 58, p.963-970, 1985.

McLAREN, A. C., PITKETHLY, D. R. The twinning microstructure and growth of amethyst quartz. Phys. Chem. Minerals, v. 8, p.128-135, 1982.
McMILLAN, P. F., HOFMEISTER, A.M. Infrared and Raman Spectroscopy. Reviews in Mineralogy, v. 18, p.99-159, 1988.

NUTTALL, R.H.D., WEIL, J.A. Two hydrogenic trapped-hole species in alpha-quartz. Solid State Commun., v. 33, p. 99-102, 1980.

O'BRIEN, M.C.M. The structure of colour centres in smoky quartz. Proc. Roy. Soc., v. A231, p. 404-414, 1955.

PASQUALI, M. A. Separação e caracterização de impurezas no quartzo natural - aplicação de métodos de lixiviação aquosa para extração de inclusões fluidas. Campinas: FEMUNICAMP, 1992. 203p. (Dissertação de Mestrado).

PATERSON, M. S. The determination of hydroxyl by infrared absorption in quartz, silicate glasses and similar materials. Bull. Mineral., v.105, p. 20-29, 1982.

SCHLOSSIN, H.H., LANG, A.R. A study of repeated twinning, lattice imperfections and impurity distribution in amethyst. Philos. Mag., v. 12, p. 283-296, 1965.

SUNAGAWA, I. Crystals: growth, morphology and perfection. Cambridge: Cambridge Universtity Press, 2005. 295p.

Artigo recebido em 16/11/2008 e aprovado em 08/04/2009.

\title{
Descubra as muitas informações da:
}

\author{
Geologia, Mineração, \\ Metalurgia \& Materiais
}

e Engenharia Civil.

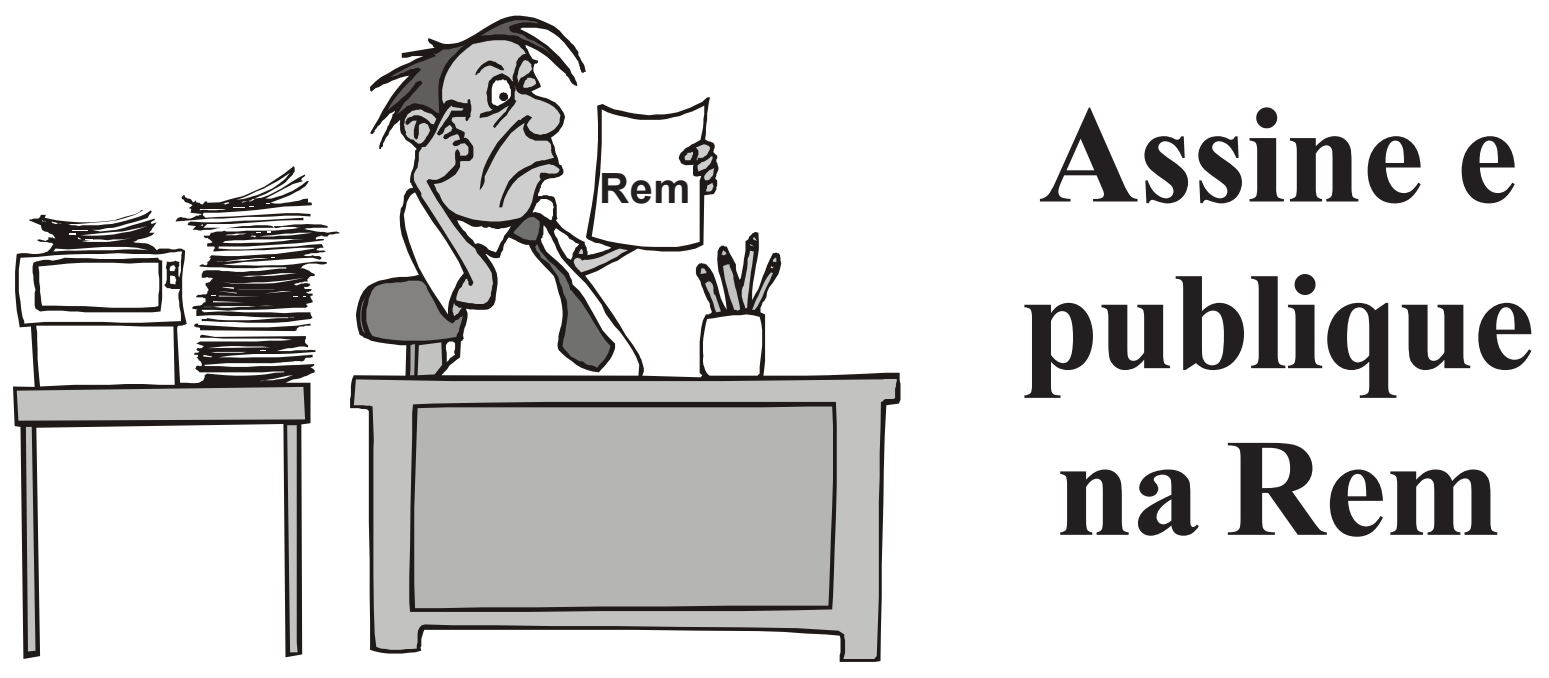

Conheça o nosso site: WWW.rem.com.br 